\title{
Outcomes of hypertonic saline inhalation as a treatment modality in nontuberculous mycobacterial pulmonary disease
}

\begin{abstract}
To the Editor:
Treatment of nontuberculous mycobacterial pulmonary disease (NTM-PD) poses a challenge for clinicians and patients. Treatment involves 18-24 months of multidrug antibiotic therapy and has a cure rate of 50$70 \%$ [1]. Cure rates depend on the causative NTM species, drug susceptibility and disease manifestation; macrolide resistance and fibro-cavitary disease are risk factors for treatment failure. Even when treatment is considered successful, there is a high risk of recurrence, either by relapse or re-infection. Furthermore, adverse events, like gastrointestinal discomfort, are frequently seen and occasionally necessitate treatment discontinuation [1]. Management of underlying pulmonary disease is essential for successful NTM-PD treatment [1]. Since hypertonic saline (HS) inhalation is safe and has shown a clinical effect in bronchiectasis patients [2-4] we have included HS inhalation in our local NTM treatment protocol. In clinical practice, we advise patients with nodular-bronchiectatic disease to start with HS inhalation two times daily as the only therapy for the first 3 months. When patients tolerate HS and when there are no clinical signs of disease deterioration, we continue HS as the only treatment with close observation of possible disease progression. This clinical observation includes a high-resolution chest tomography, sputum or bronchoalveolar lavage mycobacterial cultures and pulmonary function tests.
\end{abstract}

In an in vitro study we have observed killing of Mycobacterium avium and growth inhibition of Mycobacterium abscessus bacteria when exposed to HS (5.8\%) (figure 1a and b) (see reference [5] for a comparable methodology of exposing mycobacteria to different concentrations antibiotics). Since we were already using HS in our NTM patients, together with the known positive effects of HS in bronchiectasis patients and this in vitro observation, we decided to perform a retrospective analysis of NTM patients treated with HS.

To determine the effect of HS inhalation in NTM-PD patients we examined the medical records of all patients diagnosed with NTM-PD between January 2015 and December 2017 at our NTM expert centre. Patients who used HS inhalation were identified via medication lists and annotations in the electronic medical records. NTM-PD patients were eligible for the study if they were treated with HS inhalation with no antibiotic NTM treatment for a duration of at least 3 months. All patients that initiated HS inhalation were seen by a physiotherapist to practice active cycle of breathing technique for improving sputum clearance directly after HS inhalation. The standard nebuliser device used in our centre is the Innospire Deluxe compressor (Philips Respironics, Eindhoven, the Netherlands). Study endpoints included symptom severity, pulmonary function, microbiological and radiological parameters. Culture conversion was defined according to NTM-NET definitions: the finding of at least two consecutive negative mycobacterial cultures collected at least 4 weeks apart [6]. Radiological outcome was determined by radiologists' reports and evaluation by a pulmonologist (S.M.H. Zweijpfenning, C. Magis-Escurra and W. Hoefsloot).

We identified 25 patients (six men and 19 women) with a mean \pm sD age of $69.0 \pm 9.8$ years. Mean \pm SD body mass index was $22.2 \pm 3.5 \mathrm{~kg} \cdot \mathrm{m}^{-2}$. Most of the patients presented with nodular-bronchiectatic disease $(\mathrm{n}=19 ; 76 \%)$, five patients had fibro-cavitary disease. A history of smoking $(\mathrm{n}=17 ; 68 \%$; there were no current smokers at the time of study inclusion) and COPD comorbidity ( $n=9 ; 36 \%)$ were common. The mean \pm SD duration between the first positive mycobacterial sputum culture and start of HS inhalation was

@ERSpublications

Patients with nontuberculous mycobacterial lung disease benefit from hypertonic saline inhalation http://bit.ly/2WQpYMz

Cite this article as: Huiberts A, Zweijpfenning SMH, Pennings LJ, et al. Outcomes of hypertonic saline inhalation as a treatment modality in nontuberculous mycobacterial pulmonary disease. Eur Respir J 2019; 54: 1802143 [https://doi.org/10.1183/13993003.02143-2018]. 

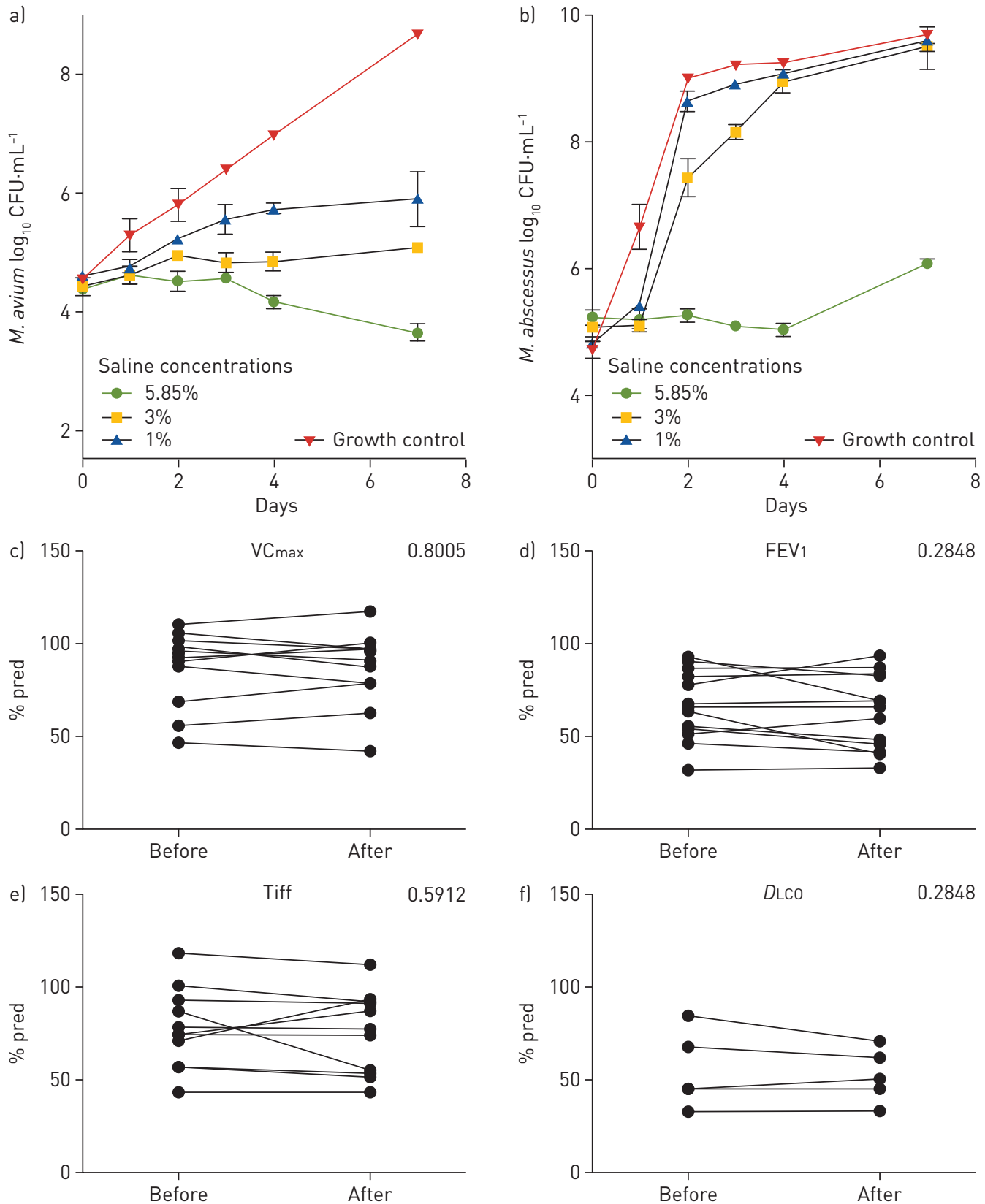

FIGURE 1 Growth curves of a) Mycobacterium avium ATCC700898 and b) Mycobacterium abscessus CIP104536 exposed to escalating concentrations of saline $(5.85 \%, 3 \%, 1 \%$ and growth control) by counting colony forming units (CFU) on day $0,1,2,3,4$ and 7 . CFU counts are the means of an experiment performed in duplicate. $\mathrm{c}-\mathrm{f}$ ) Lung function parameters (\% pred) before the start of hypertonic saline inhalation and after 3 months of hypertonic saline inhalation. c) Maximum vital capacity $\left(\mathrm{VC}_{\max }\right)$, d) forced expiratory volume in 1s ( $\left.F E V 1\right)$, e) Tiffeneau-Pinelli index (Tiff) and f) diffusing capacity of the lung for carbon monoxide (Dıco).

9.8 \pm 9.2 months (range 1-36 months). M. avium complex (MAC) bacteria were the predominant causative agents $(n=12 ; 48 \%)$. Cough $(n=23 ; 92 \%)$, sputum production $(n=20 ; 80 \%)$, fatigue $(n=9 ; 36 \%)$ and dyspnoea $(n=12 ; 48 \%)$ were the most frequently reported symptoms before start of HS inhalation. Four patients were smear positive before HS initiation. Other pathogens that were isolated were Pseudomonas aeruginosa in three patients, Aspergillus fumigatus in five patients and Haemophilus influenzae in eight patients.

After 3 months use of HS inhalation, nine (36\%) patients reported symptomatic improvement, seven (28\%) remained stable and five (20\%) patients reported deterioration of symptoms. Of these latter five patients, three experienced physical complaints (increase in cough intensity in one patient and dyspnoea 
in two patients) due to HS. Three patients (12\%) showed radiographic improvement (mainly a decrease in the size/amount of nodular infiltrates), 10 (40\%) remained stable and 8 (32\%) showed deterioration.

No significant changes in lung function parameters were measured over the first 3 months of HS therapy (figure $1 \mathrm{c}-\mathrm{f}$ ).

Of the 25 patients in this study, 17 continued HS beyond the initial 3-month trial: 12 continued HS without antibiotic treatment (seven MAC, three M. abscessus, one Mycobacterium malmoense, one Mycobacterium fortuitum and one Mycobacterium chelonae), five continued HS and started antibiotic treatment (all MAC, two fibro-cavitary disease patients). Of the remaining eight patients, three were lost to follow-up, two patients started antibiotic treatment, and three patients did not start antibiotic treatment. Of the 12 patients who continued HS without antibiotic treatment, six attained culture conversion (all nodular-bronchiectatic disease patients; two MAC, two M. abscessus, one M.fortuitum and one M. malmoense); mean \pm SD time to culture conversion $23.5 \pm 9.9$ weeks. Patients that showed no culture conversion in this group (including three patients with fibro-cavitary disease) continued HS because of improvement of symptoms. The mean \pm sD follow-up time for all patients in this study was $16.2 \pm 19.0$ months (range 3-36 months), a mean \pm SD of $5.8 \pm 5.0$ cultures from pulmonary specimens were carried out in this follow-up (mainly sputum cultures). For the six patients that showed culture conversion the mean \pm SD follow-up time was $10.8 \pm 9.9$ months. In this follow-up a mean \pm SD of $5.1 \pm 3.4$ sputum cultures were carried out.

The study cohort had too small a sample size for conclusions about the subgroups. As fibro-cavitary NTM-PD tends to be more rapidly progressive, the use of HS inhalation as a single treatment modality might be safer in nodular-bronchiectatic disease patients. Additionally, HS might be beneficial as an initial treatment option for patients with a resistant NTM species, where the risk of side-effects is not balanced with the expected effect of antibiotic treatment, or in of those with comorbidities like reflux oesophagitis or smoking, for whom antibiotic therapy is less frequently successful [6]. Since we have also observed clinical deterioration in patients treated with only HS, including side-effects from HS, a close follow-up is warranted.

The positive effect of HS in our NTM-PD cohort is probably due to the increase in mucociliary clearance of the airways. This improved clearance can be the result of direct mechanical effects of HS inhalation (improved sputum clearance by HS-induced coughing) but also the result of rehydration (as a result of an increase in the osmotic gradient) of the periciliary fluid and mucus layer improving transportability of sputum [7]. Furthermore, HS may have immunomodulatory properties. HS enhances T-helper cell (Th) 17 response in vitro [8] and has anti-inflammatory effects in cystic fibrosis patients. Although Th17 derived cytokines are associated with autoimmune diseases, they are important for mucosal and cutaneous defences. Several recent studies found that NTM-PD is associated with reduced Th17 immunity and an attenuated interleukin-17 response [9-11]. HS might therefore enhance the adaptive immune response against NTM-PD.

In conclusion, in this retrospective cohort of NTM-PD patients, HS inhalation as a single treatment modality appeared to be well tolerated and beneficial; it may even enhance sputum culture conversion. HS inhalation might be of additive value in the treatment of NTM-PD in patients with the nodular-bronchiectatic disease type, and possibly offers an opportunity to boost, postpone or obviate antibiotic treatment. As is already recommended in NTM guidelines, antibiotic treatment of NTM-PD must be considered if symptoms, radiographic abnormalities and positive NTM cultures persist after treatment of related comorbid conditions including but not limited to airway clearance for bronchiectasis. As we have observed HS-induced killing of NTM in vitro, HS inhalation can also be considered in addition to antibiotic treatment for fibro-cavitary disease. The exact effect and role of HS should be the subject of a randomised controlled trial.

Anne Huiberts ${ }^{1}$, Sanne M.H. Zweijpfenning, Lian J. Pennings ${ }^{2}$, Martin J. Boeree ${ }^{1}$, Jakko van Ingen $\odot^{2}$, Cecile Magis-Escurra ${ }^{1}$ and Wouter Hoefsloot $\oplus^{1}$

${ }^{1}$ Dept of Pulmonary Diseases, Radboud Center for Infectious diseases, Radboud University Medical Center, Nijmegen, The Netherlands. ${ }^{2}$ Dept of Medical Microbiology, Radboud Center for Infectious Diseases, Radboud University Medical Center, Nijmegen, The Netherlands.

Correspondence: Wouter Hoefsloot, Dept of Pulmonary Diseases, PO Box 9101, 6500HB Nijmegen, The Netherlands. E-mail: wouter.hoefsloot@radboudumc.nl

Received: Nov 092018 | Accepted after revision: April 072019

Conflict of interest: None declared. 


\section{References}

1 Zweijpfenning S, Kops S, Magis-Escurra C, et al. Treatment and outcome of non-tuberculous mycobacterial pulmonary disease in a predominantly fibro-cavitary disease cohort. Respir Med 2017; 131: 220-224.

2 Kellett F, Redfern J, Niven RM. Evaluation of nebulised hypertonic saline (7\%) as an adjunct to physiotherapy in patients with stable bronchiectasis. Respir Med 2005; 99: 27-31.

3 Kellett F, Robert NM. Nebulised 7\% hypertonic saline improves lung function and quality of life in bronchiectasis. Respir Med 2011; 105: 1831-1835.

4 Wark P, Mcdonald VM. Nebulised hypertonic saline for cystic fibrosis. Cochrane Database Syst Rev 2009; 2: CD001506.

5 Ruth MM, Sangen JJN, Remmers K, et al. A bedaquiline/clofazimine combination regimen might add activity to the treatment of clinically relevant non-tuberculous mycobacteria. J Antimicrob Chemother 2019; 74: 935-943.

6 Van Ingen J, Aksamit T, Andrejak C, et al. Treatment outcome definitions in nontuberculous mycobacterial pulmonary disease: an NTM-NET consensus statement. Eur Respir J 2018; 51: 1800170.

7 Willis PJ, Hall RL, Chan W, et al. Sodium chloride increases ciliary transportability of cystic fibrosis and bronchiectasis sputum on the mucus depleted bovine trachea. J Clin Invest 1997; 99: 9-13.

8 Van Der Meer JWM, Netea MG. A salty taste to autoimmunity. N Engl J Med 2013; 368: 2520-2521.

9 Lim A, Allison C, Price P, et al. Susceptibility to pulmonary disease due to Mycobacterium avium-intracellulare complex may reflect low IL-17 and high IL-10 responses rather than Th1 deficiency. Clin Immunol 2010; 137: 296-302.

10 Shu CC, Wang JY, Wu MF, et al. Interleukin 23/interleukin 17 axis activated by Mycobacterium avium complex (MAC) is attenuated in patients with MAC-lung disease. Tuberculosis (Edinb) 2018; 110: 7-14.

11 Becker KL, Van Ingen J, Ten Oever J, et al. Deficient interleukin-17 production in response to Mycobacterium abscessus in cystic fibrosis. Eur Respir J 2016; 47: 990-993. 